\title{
A multifunctional CT technology: Reality or illusion for patient risk assessment?
}

\author{
Tomoaki Nakata, MD, PhD ${ }^{a, b}$ \\ a Department of Cardiology, Hakodate Goryoukaku Hospital, Hakodate, Japan \\ b Sapporo Medical University School of Medicine, Sapporo, Japan
}

Received Feb 1, 2016; accepted Feb 1, 2016

doi:10.1007/s12350-016-0439-x

\begin{abstract}
A high-speed, multi-slice coronary computed tomography (CT) imaging has emerged as a promising or clinically available multifunctional technique for the assessment of myocardial ischemia, viability, ischemia-induced cardiac dysfunction, and coronary atherosclerotic alterations in patients with suspected or known coronary artery disease. Despite several technical issues remain to be resolved, cardiac CT imaging will have a reality as a multifunctional modality for guiding physicians in better decision-making for favorable clinical outcomes in patients with suspected coronary artery disease, provided that this imaging technology can contribute to characterization and localization of high-risk coronary atherosclerosis in combination with the quantitative evaluation of functional myocardial ischemia.
\end{abstract}

Key Words: Myocardial ischemia $\cdot$ stree myocardial perfusion imaging $\cdot$ coronary artery disease $\cdot$ cardiac CT

\section{See related article, pp. 1253-1262}

Several non-invasive modalities have emerged as promising or clinically available multifunctional techniques for the assessment of myocardial ischemia, ischemic burden size, ischemia-induced left ventricular dysfunction, myocardial viability, and high-risk coronary plaque, all of which are definitively related to unfavorable clinical outcomes in patients with suspected or known coronary artery disease. Among them, recent remarkable advances in high-speed, multi-slice computed tomography (CT) in cardiac imaging enable to evaluate not only coronary arteriosclerosis, including luminal narrowing, vulnerable plaque, and atherosclerotic remodeling, but also functional ischemia by the measurement of coronary flow reserve with or without a vasodilator agent. A wide-spread use and easy access to

Reprint requests: Tomoaki Nakata, MD, PhD, Department of Cardiology, Hakodate Goryoukaku Hospital, Hakodate, 040-8611, Japan; tnakata@sapmed.ac.jp

J Nucl Cardiol 2017;24:1263-6.

$1071-3581 / \$ 34.00$

Copyright (C) 2016 American Society of Nuclear Cardiology. coronary $\mathrm{CT}$ at cardiology practice contributes to identifying cryptogenic coronary arteriosclerosis and vulnerable coronary plaque. On the other hand, anatomical approach to coronary artery disease tends to overestimate coronary luminal narrowing, which is not necessarily the hallmark of significant coronary artery disease or future cardiac event risks, ${ }^{1-3}$ and sometimes results in unnecessary or ineffective selection of invasive diagnostic or therapeutic strategy. ${ }^{4-10}$ In this context, cardiac CT technology as a multifunctional modality is still challenging and in progress. In this issue of $J$ Nucl Cardiol, van Rosendael et al ${ }^{11}$ investigated the association between atherosclerotic coronary morphology and functional ischemia both of which were estimated on the same day using a sequential CT imaging protocol together with an adenosine stress approach in 84 of 115 consecutive patients with new onset chest pain referred from the outpatient clinic. Authors demonstrated a limited but positive correlation between CT angiographic stenosis and adenosine-induced CT perfusion abnormality. Besides the small, single-center study and the limited number of ischemic lesions, there are several issues to be recognized in this study before these results can be put into clinical perspective. 
Firstly, the presented study showed that coronary stenosis percentage quantitatively assessed was moderately related to myocardial ischemia but lumen area stenosis and lesion length were independently associated with ischemia when adjusted for coronary plaque volume, mean plaque burden, maximal lesion thickness, and dense calcium volume. Although authors tried to correlate the atherosclerotic changes of coronary artery morphology, other than luminal narrowing, with CT perfusion abnormality, the clinical implications are unclear in this study. Coronary CT angiography has high negative predictive but limited positive predictive values (that is, high false positive values) in the detection of significant coronary artery stenosis. ${ }^{1,2,12,13}$ In addition, the coarse classification of luminal stenosis into four groups $(\leq 40 \%, 41-60 \%, 61-80 \%$, and $81 \%$-) directly indicates technical limitations of coronary CT angiography and the results also showed the limited association of $61-80 \%$ and $>81 \%$ stenoses with inducible ischemia ( $34 \%$ and $59 \%$, respectively) defined as a summed difference score of $\geq 1$ on stress CT perfusion imaging. These findings, however, are not uncommon because many studies have shown discrepancy between the severity of coronary CT-defined stenosis and inducible myocardial ischemia or ischemia-producing coronary stenosis identified by non-invasive and invasive methods. Nevertheless, the presented findings strongly suggest that pathophysiological roles of coronary atherosclerotic alterations are modified by multiple morphological features in a complicated manner but not simply determined by the severity of coronary liminal narrowing as previously believed for decades.

Secondly, a lack of a gold standard of functional myocardial ischemia such as fractional flow reserve assessed by an invasive technique or coronary flow reserve quantified by positron $\mathrm{CT}$ imaging attenuates the clinical values of the presented correlations between morphological CT findings and myocardial CT perfusion abnormality. George RT et $\mathrm{al}^{14}$ recently demonstrated the overall better sensitivity of adenosine stress myocardial CT perfusion imaging when directly compared to scintigraphic stress myocardial perfusion imaging in the diagnosis of anatomical coronary stenosis defined as $\geq 50 \%$ by invasive quantitative coronary angiography. The data, however, showed that stress CT perfusion imaging has lower positive predictive values and lower specificities at each coronary territory due to false positive results. As stated, because of limitations of coronary morphology by coronary CT angiography for ischemia detection, integration of coronary CT angiography with the assessment of myocardial ischemia in one session is reasonable and, therefore, coronary and perfusion CT information should be complementarily utilized in a clinical setting. The clinical implications of the presented findings, however, basically depend on the diagnostic accuracy of adenosine-stress CT perfusion imaging in the detection of functional myocardial ischemia and patient selection based on clinically acceptable criteria. There is robust evidence that myocardial burden size of functional myocardial ischemia and ischemia-related left ventricular dysfunction both of which are assessed simultaneously by stress electrocardiogram-gated myocardial single-photon emission computed tomography have mid- to long-term cardiac events. ${ }^{15-18}$ Together with the issue previously mentioned, two types of non-negligible false positive features in the assessment of anatomical narrowing by coronary $\mathrm{CT}$ angiography and functional ischemia by stress myocardial CT perfusion imaging have to be furthermore overcome for the improvement in the clinical values of the presented findings so that their complementary assessment using a contrast medium and a stress agent could be more effective in the clinical setting.

Thirdly, anatomical CT results were not blinded to physicians who subsequently evaluated stress perfusion CT data, indicating the possibility of diagnostic bias to more correlate abnormal $\mathrm{CT}$ morphologies with myocardial CT perfusion results. The presented findings show that plaque assessment itself is unlikely to be related to coronary luminal narrowing but may contribute to the improvement in the detection of functional ischemia by adenosine-stress CT perfusion imaging. Besides the diagnostic efficacy in the ischemia detection, there is a multiplicity of clinical implications of cardiac CT characterizations of atherosclerotic plaque, inflammatory reactions, and composition of coronary artery wall, possibly contributing to the improvement in the prediction of short-term to long-term cardiovascular risk assessment in patients presenting with acute chest pain or suspected coronary artery disease. ${ }^{19-21}$ Acute coronary syndromes definitively result from vulnerable plaque rupture followed by sudden thrombus obstruction of coronary artery, relating to distinct morphological alterations of coronary artery such as low-density, lipidrich necrotic core covered with a thin fibrous cap and spotty or micro-calcification. ${ }^{22,23}$ These findings, which may be able to be depicted more clearly by positron emission tomography with fluorine 18-fluorodeoxyglucose or cardiac magnetic resonance imaging compared to coronary CT imaging, ${ }^{21-24}$ are likely to be a more immediate or short-term risk indicator of adverse outcomes. On the other hand, Cho et al ${ }^{25}$ recently showed incremental prognostic values of the assessment of atherosclerotic plaque burden and lumen narrowing by coronary CT angiography in asymptomatic patients with moderate coronary calcification defined as calcium score ranging from 101 to 400 during a 24 -month period. The 
prognostic efficacy, however, is likely to be attenuated ironically in patients with lower or higher coronary calcification probably due to false positive or negative results in the presence of severe calcification and/or motion artifacts. Thus, it may be more valuable to separately correlate various morphological alterations of coronary artery, such as luminal stenosis, coronary plaque characteristics (the number, volume, length, and location of plaque), the degrees of covered fibrous cap, and calcium deposition and coronary remodeling with inducible myocardial ischemia from not only pathophysiological or diagnostic but also prognostic points of view.

Finally, $31(27 \%)$ patients were excluded from the analysis, because of insufficient image quality, previous coronary interventional treatment, anomalous coronary arteries, and the presence of a fixed CT perfusion defect. These limitations in routine clinical application of cardiac CT imaging are likely to be originated in imaging techniques such as insufficient heart rate control, motion artifacts, limited spatial and contrast resolutions, and anatomical approach per se. In addition to advanced impairment of kidney function, high-risks for contrastinduced nephropathy or allergic reactions to contrast medium, these clinical situations to which the cardiac CT imaging is less applicable are not uncommon at daily practice and in an urgent situation and, therefore, have to be considered more sufficiently for patient safety and better cost-effectiveness of this procedure.

Thus, despite several issues remain to be resolved, this article provides clinically important suggestions with an emphasis on characterizations of coronary atherosclerosis in association with functional impairment of coronary flow reserve. Myocardial ischemia assessed by adenosine stress CT perfusion imaging is likely to depend not only on luminal stenosis but also on the quantity of coronary plaque and calcium deposition. The pathological implications of these complicated features, however, may vary in each coronary territory in each patient. Beyond a diagnostic triage tool, cardiac CT imaging will have a reality as a multifunctional modality for guiding physicians in an appropriate selection of preventive or therapeutic strategy for better clinical outcomes in patients with new onset chest pain or suspected coronary artery disease, provided that this imaging technology can contribute to characterization and localization of high-risk coronary atherosclerosis in combination with the quantitative evaluation of functional myocardial ischemia.

\section{Disclosure}

Tomoaki Nakata has no conflict of interest to be declared.

\section{References}

1. Leber AW, Knez A, von Ziegler F, Becker A, Nikolaou K, Paul S, et al. Quantification of obstructive and nonobstructive coronary lesions by 64-slice computed tomography: A comparative study with quantitative coronary angiography and intravascular ultrasound. J Am Coll Cardiol 2005;46:147-54.

2. Nallamothu BK, Spertus JA, Lansky AJ, Cohen DJ, Jones PG, Kureshi F, et al. Comparison of clinical interpretation with visual assessment and quantitative coronary angiography in patients undergoing percutaneous coronary intervention in contemporary practice: The Assessing Angiography (A2) project. Circulation 2013;127:1793-800.

3. Gebhard C, Fuchs TA, Stehli J, Gransar H, Berman DS, Budoff $\mathrm{MJ}$, et al. Coronary dominance and prognosis in patients undergoing coronary computed tomographic angiography: results from the CONFIRM (COronary CT Angiography EvaluatioN For Clinical Outcomes: An InteRnational Multicenter) registry. Eur Heart J Cardiovasc Imaging 2015;16:853-62.

4. Boden WE, O'Rourke RA, Teo KK, Hartigan PM, Maron DJ, Kostuk WJ, et al. Optimal medical therapy with or without PCI for stable coronary disease. N Engl J Med 2007;356:1503-16.

5. BARI 2D Study Group, Frye RL, August P, Brooks MM, Hardison $\mathrm{RM}$, Kelsey SF, et al. A randomized trial of therapies for type 2 diabetes and coronary artery disease. N Engl J Med 2009;360:2503-15.

6. Tonino PA, De Bruyne B, Pijls NH, Siebert U, Ikeno F, van' t Veer M, et al. Fractional flow reserve versus angiography for guiding percutaneous coronary intervention. $\mathrm{N}$ Engl $\mathrm{J}$ Med 2009;360:213-24

7. Pijls NH, Fearon WF, Tonino PA, Siebert U, Ikeno F, Bornschein $\mathrm{B}$, et al. Fractional flow reserve versus angiography for guiding percutaneous coronary intervention in patients with multivessel coronary artery disease: 2-year follow-up of the FAME (Fractional Flow Reserve Versus Angiography for Multivessel Evaluation) study. J Am Coll Cardiol 2010;56:177-84.

8. Douglas PS, Hoffmann U, Patel MR, Mark DB, Al-Khalidi HR, Cavanaugh B, et al. Outcomes of anatomical versus functional testing for coronary artery disease. N Engl J Med 2015;372:1291300 .

9. Shreibati JB, Baker LC, Hlatky MA. Association of coronary CT angiography or stress testing with subsequent utilization and spending among Medicare beneficiaries. JAMA 2011;306:212836.

10. Yamauchi T, Tamaki N, Kasanuki H, Kimura T, Uemura Y, Iimuro $\mathrm{S}$, et al. Optimal initial diagnostic strategies for the evaluation of stable angina patients: A multicenter, prospective study on myocardial perfusion imaging, computed tomographic angiography, and coronary angiography. Circ J 2012;76:2832-9.

11. van Rosendael AR, Kroft LJ; Broersen A, Dijkstra J, van den Hoogen IJ, van Zwet EW et al. Relation between quantitative coronary CTA and myocardialischemia by adenosine stress myocardial CT perfusion. J Nucl Cardiol 2016; 80 (in press).

12. Doh JH, Koo BK, Nam CW, Kim JH, Min JK, Nakazato R, et al. Diagnostic value of coronary $\mathrm{CT}$ angiography in comparison with invasive coronary angiography and intravascular ultrasound in patients with intermediate coronary artery stenosis: Results from the prospective multicentre FIGURE-OUT (Functional Imaging criteria for GUiding REview of invasive coronary angiOgraphy, intravascular Ultrasound, and coronary computed Tomographic angiography) study. Eur Heart J Cardiovasc Imaging 2014;15:8707.

13. Kim HY, Choi JH. How to utilize coronary computed tomography angiography in the treatment of coronary artery disease. J Cardiovasc Ultrasound 2015;23:204-8. 
14. George RT, Mehra VC, Chen MY, Kitagawa K, Arbab-Zadeh A, Miller JM, et al. Myocardial CT perfusion imaging and SPECT for the diagnosis of coronary artery disease: A head-to-head comparison from the CORE320 multicenter diagnostic performance study. Radiology 2014;272:407-16.

15. Sharir T, Germano G, Kavanagh PB, Lai S, Cohen I, Lewin HC, et al. Incremental prognostic value of post-stress left ventricular ejection fraction and volume by gated myocardial perfusion single photon emission computed tomography. Circulation 1999;100: 1035-42.

16. Shaw LJ, Berman DS, Maron DJ, Mancini GB, Hayes SW, Hartigan PM, et al. Optimal medical therapy with or without percutaneous coronary intervention to reduce ischemic burden: Results from the Clinical Outcomes Utilizing Revascularization and Aggressive Drug Evaluation (COURAGE) trial nuclear substudy. Circulation 2008;117:1283-91.

17. Nakata T, Hashimoto A, Wakabayashi T, Kusuoka H, Nishimura T. Prediction of new-onset refractory congestive heart failure using stress/rest gated perfusion SPECT imaging in patients with known or suspected coronary artery disease: Sub-analysis of the JACCESS. J Am Coll Cardiol Cardiovasc Imaging 2009;2:1393400.

18. Shaw LJ, Cerqueira MD, Brooks MM, Althouse AD, Sansing VV, Beller GA, et al. Impact of left ventricular function and the extent of ischemia and scar by stress myocardial perfusion imaging on prognosis and therapeutic risk reduction in diabetic patients with coronary artery disease: Results from the Bypass Angioplasty
Revascularization Investigation 2 Diabetes (BARI 2D) trial. J Nucl Cardiol 2012;19:658-69.

19. Alexopoulos N, Raggi P. Calcification in atherosclerosis. Nat Rev Cardiol 2009;6:681-8.

20. Voros S, Rinehart S, Qian Z, Joshi P, Vazquez G, Fischer C, et al. Coronary atherosclerosis imaging by coronary $\mathrm{CT}$ angiography: Current status, correlation with intravascular interrogation and meta-analysis. JACC Cardiovasc Imaging 2011;4:537-48.

21. Korosoglou G, Giusca S, Gitsioudis G, Erbel C, Katus HA. Cardiac magnetic resonance and computed tomography angiography for clinical imaging of stable coronary artery disease. Diagnostic classification and risk stratification. Front Physiol 2014;5:291. doi: 10.3389/fphys.2014.00291 eCollection 2014.

22. Falk E, Shah PK, Fuster V. Coronary plaque disruption. Circulation 1995;92:657-71.

23. Stolzmann P, Subramanian S, Abdelbaky A, Maurovich-Horvat P, Scheffel H, Tawakol A, et al. Complementary value of cardiac FDG PET and CT for the characterization of atherosclerotic disease. Radiographics 2011;31:1255-69.

24. Corti R, Fuster V. Imaging of atherosclerosis: Magnetic resonance imaging. Eur Heart J 2011;32:1709-19.

25. Cho I, Chang HJ, ÓHartaigh B, Shin S, Sung JM, Lin FY, et al. Incremental prognostic utility of coronary $\mathrm{CT}$ angiography for asymptomatic patients based upon extent and severity of coronary artery calcium: results from the COronary CT Angiography EvaluatioN For Clinical Outcomes InteRnational Multicenter (CONFIRM) study. Eur Heart J 2015;21:501-8. 